\title{
MEDICINA BASADA EN EVIDENCIAS PARA MEJORAR COMPETENCIAS EN EL SEGUIMIENTO FARMACOTERAPÉUTICO
}

\author{
Evidence based medicine for improving skills in pharmacotherapy follow up
}

Gladys M. Delgado-Pérez ${ }^{1,3}$, Roselly M. Robles ${ }^{2}$, Julio C. Rodríguez ${ }^{3}$, Eloisa M. Hernández ${ }^{1}$, Doris H. Delgado-Pérez ${ }^{4}$

${ }^{1}$ Facultad de Farmacia y Bioquímica, Universidad Nacional Mayor de San Marcos. ${ }^{2}$ Centro Nacional de Información de Medicamentos, Dirección General de Medicamentos Insumos y Drogas, Lima-Perú. ${ }^{3}$ Hospital PNP Luis N. Saenz, ${ }^{4}$ Facultad de Medicina, Universidad Nacional Mayor de San Marcos

\section{RESUMEN}

En el aprendizaje del Seguimiento Farmacoterapéutico (SFT), uno de los aspectos es evaluar en forma correcta los datos clínicos del paciente y los medicamentos que consume, y se logre que cada decisión a tomar, sea sustentada por una investigación que demuestre ser la más adecuada desde una perspectiva de racionalidad. El objetivo de este estudio fue determinar la mejora de las competencias de los estudiantes de pre-grado en el Seguimiento Farmacoterapéutico, en la búsqueda de información científica de medicamentos aplicando la MBE. Se aplicó un diseño analítico, cuasi-experimental, prospectivo, longitudinal desarrollado en dos etapas: primero con la metodología tradicional y luego con la MBE, estructurando preguntas clínicas mediante la estrategia PICO. Con la MBE se recuperaron $74 \%$ de literaturas de mayor calidad de evidencia (metanálisis, ensayos clínicos, estudios de cohortes, serie de casos, opinión de expertos), frente a lo recuperado con la metodología tradicional (26\%), que recuperó principalmente revisiones sistemáticas. Las competencias para detectar Problemas Relacionados a Medicamentos (PRM), cuando se aplicó la MBE, fueron de $58 \%$, frente al $42 \%$ con el método tradicional; asimismo, las competencias para plantear intervenciones farmacéuticas con el método de la MBE fue de 55\% frente al 45\% con la tradicional. En conclusión, los estudiantes lograron mejorar las competencias en la detección de PRM en un $20 \%$ mientras que en la intervención farmacéutica en $8 \%$.

Palabras clave: Estrategia PICO, efectividad y seguridad de medicamentos, seguimiento farmacoterapéutico.

\section{SUMMARY}

In learning Pharmacotherapy Follow up (PF), an aspect is to assess correctly the clinical patient data and consuming drugs, and applying the "Evidence Based Medicine" (EBM), it is achieved that every decision to take, be supported by research that proves to be the most appropriate from the perspective of rationality. The aim of this study was to improve the skills of undergraduate students in the Pharmacotherapy Follow up, in the search for scientific drug information using the EBM. It is a quasi-experimental, prospective, longitudinal analytical design, developed in two stages: first with the traditional methodology and then with the EBM, structuring clinical questions through the PICO strategy. With the EBM were recovered $74 \%$ of literatures higher quality of evidence (meta-analysis, clinical trials, cohort studies, case series, expert opinion), against to recoveries with the traditional methodology (26\%), recovering_systematic reviews mainly. The effectiveness to detect DrugRelated Problems (DRP), when EBM was applied, was $58 \%$, versus $42 \%$ with traditional method; besides, the effectiveness to raise pharmaceutical interventions with EBM method was $55 \%$ versus $45 \%$ with the traditional. Was concluded that students managed to improve skills detecting DRP in $20 \%$ and while in the pharmaceutical intervention in $8 \%$.

Keywords: PICO strategy, efffectiveness and security of drugs, pharmacotherapy follow up.

\section{INTRODUCCIÓN}

$E^{\prime}$

el aprendizaje del Seguimiento Farmacoterapéutico(SFT) unodeloseslabones más importantes es evaluar en forma correcta los datos clínicos del paciente y los medicamentos que consume ${ }^{(1,2)}$, para lo cual se puede aplicar la "Medicina Basada en Evidencias" (MBE), la cual establece que cada decisión a tomar, debe estar sustentada por una investigación que demuestre que aquélla es la más adecuada desde una perspectiva de racionalidad. Esta metodología ayuda a enseñar y evaluar al estudiante ${ }^{(3-5)}$. Existen diversas estrategias para la búsqueda de la mejor evidencia en bibliotecas virtuales, sin embargo los iniciadores de la corriente de la MBE recomiendan seguir la estrategia PICO, (P) el paciente y/o problema de interés, (I) la intervención principal, que usualmente es un tratamiento nuevo, (C) la comparación o el control, O (outcome) el resultado o desenlacedeinterés ${ }^{(6)}$. Estoscuatroelementosson básicosen la formulación apropiada de una pregunta estructurada que permita delimitar o precisar la necesidad de la información y hacer más fácil la búsqueda. En el estudio se plantea 
identificar fuentes de información que faciliten el análisis correcto en la resolución de los Problemas Relacionados con el Medicamento (PRM), logrando seleccionar estudios con mayor calidad de evidencia, como son los estudios de meta-análisis, revisiones sistemáticas y ensayos clínicos controlados, con los cuales se conseguirá mejorar el análisis de un caso clínico ${ }^{(7)}$.

La integración del aprendizaje activo -en el silabo de Atención Farmacéutica-, ha sido identificada como estrategia inicial para mejorar los conocimientos, las habilidades en un aprendizaje autodirigido, y la promoción de habilidades para resolver problemas. El aprendizaje basado en problemas (ABP) es una técnica con un enfoque de aprendizaje activo dirigido al estudiante. Durante el ABP, los estudiantes desarrollan habilidades para resolver problemas, formulan decisiones basadas en la evidencia y mejoran sus habilidades de comunicación, que son esenciales para el logro de las competencias básicas. Las competencias adicionales incluyen la mejora del aprendizaje dirigido de manera independiente o en equipo, el desarrollo de la capacidad de búsqueda y evaluación de literatura, así como la aplicación de esta información en casos clínicos reales ${ }^{(8)}$.

En la asignatura de Atención Farmacéutica, se analiza y comprende la metodología del SFT, que se ejecuta mediante el desarrollo de actividades de forma sistemática, para aplicarlas en pacientes del ámbito hospitalario y ambulatorio. Dichas actividades son: selección de pacientes, obtención de los datos de salud, evaluación, análisis e identificación del PRM, desarrollo de planes de Intervención Farmacéutica. De esta forma el alumno podrá desempeñarse en los dos ámbitos, identificando, previniendo y resolviendo PRM.

El objetivo general fue aplicar la MBE para mejorar las competencias de los estudiantes de pre-grado de farmacia en la búsqueda de información científica de medicamentos. Los objetivos específicos fueron: (1) Evaluar la calidad del material recuperado antes y después de aplicar la metodología de la MBE, (2) Detallar las fuentes de información utilizadas por los estudiantes, (3) Determinar los problemas correctos e incorrectos relacionados a la efectividad y seguridad de los medicamentos, que identifiquen los estudiantes antes y después de aplicar la metodología de la MBE.

\section{MATERIALES Y MÉTODOS}

Participaron en el estudio 65 estudiantes de ambos géneros (29 mujeres y 36 varones), quienes asistieron al curso de Atención Farmacéutica en el II Semestre del año 2012. El promedio ponderado de los estudiantes fue 13,21 puntos, con una desviación estándar de o,68; lo cual nos muestra poca variabilidad en este indicador.

El estudio se realizó en dos etapas.

\section{Primera etapa}

Seaplicó la metodología tradicional donde se obtuvo la línea de base; se desarrolló durante las tres primeras semanas.

Primera semana: con el caso clínico de un paciente hospitalizado; se reconocieron los criterios de selección del paciente y el procedimiento para registrar los datos del paciente.

Segunda semana: en el aula de telemática; se presentaron las bases de datos y motores de búsqueda relacionados a medicamentos, útiles para evaluar los datos del caso clínico planteado.

Tercera semana: presentaron los PRM identificados y las Intervenciones Farmacéuticas planteadas en relación al caso clínico entregado, lo cual sirvió como nuestra línea de base.

\section{Segunda etapa}

Se utilizó la estrategia PICO para la búsqueda científica en internet y mejorar competencias en la evaluación de efectividad y seguridad de medicamentos en el SFT; el estudiante integra conocimientos de epidemiología clínica, bioestadística, métodos de investigación y de informática en la atención de la salud. El proceso involucró cuatro etapas secuenciales: la formulación de una pregunta estructurada basada en un problema clínico, la verificación de las pruebas pertinentes a partir de la literatura, la valoración crítica de la validez de la investigación contemporánea y la aplicación de los resultados para la toma de decisiones clínicas.

Una pregunta estructurada PICO se compone de cuatro elementos: $(\mathrm{P})$ problema, (I) intervención, $(\mathrm{C})$ comparación y $(\mathrm{O})$ resultados ${ }^{(4-6,9,10)}$.

Se utilizó la metodología inductiva-deductiva, analítica, donde el alumno participó en forma activa, grupal, en pares e individual, con casos reales del Hospital de Policía, logrando interactuar con los pacientes. Se aplicaron recursos didácticos para promover un aprendizaje activo participativo. Algunos de estos fueron: el $\mathrm{ABP}$, trabajos o dinámicas grupales, espacios reales de socialización, estudio de casos y trabajos individuales. Finalmente, identificaron los PRM en el paciente y propusieron Intervención Farmacéutica.

Esta segunda etapa fue desarrollada en las cuatro siguientes semanas del curso.

Cuarta semana: se formaron pares de estudiantes, quienes asistieron al Servicio de Medicina Interna 
del Hospital de Policía, donde se les facilitaron las Historias Clínicas, contando con un tutor por cada 4 pares. Lograron seleccionar a un paciente de acuerdo a criterios pre-establecidos (pacientes con medicamentos de estrecho margen terapéutico, los que tengan insuficiencia renal o hepática, los que reciben medicamentos de extrema toxicidad, los sometidos a medicación múltiple, pacientes cuyo estado clínico exige la evaluación y manipulación continua de la farmacoterapia, los que presentan algún PRM). Luego de conseguir los datos necesarios, los registraron en la ficha de Anamnesis Farmacológica, la cual fue entregada a los tutores al concluir la clase en el hospital.

Quinta semana: en el aula de informática de la Facultad, los estudiantes realizaron la búsqueda de información de medicamentos, utilizando la estrategia PICO, y aplicando de la técnica del ABP.

Sexta semana: nuevamente en el aula de telemática, realizaron la actividad guiada del análisis del caso del paciente hospitalizado, utilizando la estrategia $\mathrm{PICO}^{(6}$, ${ }^{9)}$, mediante las siguientes actividades:

a. Con el caso clínico del paciente hospitalizado identificaron los elementos de la estrategia PICO.

b. Elaboraron la(s) preguntas clínica(s) estructurada(s) utilizando los elementos identificados en el ítem anterior.

c. Establecieron el tipo de pregunta(s) clínica(s), tipos de estudio y fuentes de información.

d. Formularon la(s) estrategia(s) de búsqueda haciendo uso de los operadores booleanos (AND, OR, NOT, etc.).

e. Seleccionaron y clasificaron los artículos recuperados en las diferentes bases de datos de acuerdo a las necesidades del paciente.

f. Finalmente completaron una ficha donde señalaron la pregunta clínica, los motores de búsqueda o bases de datos, así como las literaturas recuperadas.

Entregaron toda esta información al tutor en medio electrónico.

Participaron en la enseñanza y evaluación dos Químicos Farmacéuticos del Centro Nacional de Información de Medicamentos de la Dirección General de Medicamentos Insumos y Drogas

Al final de esta semana identificaron los PRM en el paciente y propusieron la Intervención Farmacéutica, tanto para el equipo de salud como para el paciente.

Finalmente se identificaron los PRM en el paciente y propusieron la Intervención Farmacéutica, tanto para el equipo de salud como para el paciente.
Séptima semana: sustentaron los casos clínicos, y los sometieron a discusión del aula, los estudiantes participaron con preguntas relacionadas a efectividad y seguridad, la defensa del caso fue reforzada con la evidencia encontrada. Estuvieron presentes cuatro jurados externos especialistas en Farmacia Clínica y con experiencia en SFT, a quienes se les facilitó una rúbrica para la calificación tanto del análisis del caso como de la Intervención Farmacéutica planteada por los estudiantes.

Para la clasificación de las fuentes de información se utilizó la forma tradicional: primarias, secundarias y terciarias ${ }^{(6)}$; y la clasificación de Haynes ${ }^{(6)}$, según el modelo piramidal de las “5 S” (Studies, Syntheses, Synopses, Summaries y Systems).

Para evaluar los PRM de efectividad, se compararon los beneficios de los medicamentos utilizados por el paciente y la literatura encontrada. Asimismo, para los PRM de seguridad, se compararon las interacciones medicamentosas, contraindicaciones y reacciones adversas de los medicamentos utilizados por el paciente, con la literatura encontrada ${ }^{(1,2,11)}$.

\section{RESULTADOS}

Se observa que con la metodología de MBE, el mayor porcentaje de fuentes de información recuperadas por los estudiantes fueron las primarias y terciarias, mientras que con el método tradicional fueron las terciarias en su mayoría. Sin embargo, la MBE permitió ubicar el 76\% (294) de las referencias, frente al 24\% (93) del método tradicional (tabla 1).

Asimismo, el mayor porcentaje de fuentes de información recuperadas por los estudiantes, según la clasificación Haynes, fueron los estudios originales por el método MBE, mientras que con el tradicional fueron los sumarios o compendios; por otro lado, con la MBE ubicaron $83,7 \%$ (247) de las referencias, frente a 16,3\% (48) con el otro (tabla 2).

En cuanto al tipo de estudio, se observó que según el método MBE el mayor porcentaje recuperado correspondió a las guías de práctica clínica, mientras que con el método tradicional fueron los textos. Se evidenció, asimismo, que los diseños de investigación que presentaron mayor calidad de evidencia como, metaanálisisy estudios decohortes, fueron recuperados solo con el método MBE (tabla 3).

En todos los casos, con el método tradicional, un mayor porcentaje de estudiantes no identificaron estudio alguno, mientras con el método MBE, se dieron la mayoría de hallazgos, en los rubros de 4 a más de 6 
Tabla 1. Distribución porcentual de las fuentes de información recuperadas por los estudiantes según el método Tradicional y el Método MBE (n=387).

\begin{tabular}{lccccc}
\hline \multirow{2}{*}{$\begin{array}{l}\text { Tipo de fuente } \\
\text { de información }\end{array}$} & \multicolumn{2}{c}{ Tradicional } & \multicolumn{2}{c}{ MBE } & \multirow{2}{*}{ TOTAL } \\
\cline { 2 - 5 } Primaria & $\mathrm{n}$ & $\%$ & $\mathrm{n}$ & $\%$ & \\
Secundaria & 18 & 4,5 & 118 & 30,5 & \\
Terciaria & 4 & 1,1 & 2 & 0,5 & \\
Total & 71 & 18,4 & 174 & 45 & \\
\hline
\end{tabular}

evidencias (tabla 4). Estos resultados permiten afirmar que el método MBE, ayudó a conseguir mayor cantidad de estudios con diseños de investigación de mejor calidad.

En la tabla 5 se permite interpretar que la MBE fue competente en un $58 \%$ para detectar PRM, frente al $42 \%$ del método tradicional; en cuanto al hallazgo de PRM de seguridad, MEB obtuvo el 59\% y el tradicional $41 \%$; finalmente, el método tradicional detectó el 52\% de los PRM de efectividad, mientras que con MBE se encontró el $48 \%$, sin embargo en todos los casos se identificaron más PRM correctos con la MBE.

\section{DISCUSIÓN}

La estrategia de enseñanza utilizada mediante la MBE, ha sido empleada para mejorar la búsqueda y el uso de las fuentes de información ${ }^{(12)}$ y ha permitido mejorar la evaluación de la eficacia y la seguridad de los medicamentos.

Dado el incremento de enfermedades crónicas en nuestra sociedad, con la consecuente prescripción de más de tres medicamentos, y siendo una de las competencias del profesional químico farmacéutico el realizar SFT, es necesario que estas competencias clínicas se involucren desde su formación en pregrado para que logren realizar la búsqueda de información de medicamentos con alto grado de evidencia.

Asimismo, al identificar los PRM con mayor evidencia, se logra un uso realmente adecuado de los medicamentos, evitándose complicaciones y/o disminución de la estancia hospitalaria.

Elizondo ${ }^{(13)}$, manifiesta que si bien la Internet se ha convertido
Tabla 2. Distribución porcentual de las fuentes de información según la clasificación Haynes utilizadas por los estudiantes en el método Tradicional y el Método MBE. (n=295).

\begin{tabular}{|c|c|c|c|c|c|}
\hline \multirow{2}{*}{$\begin{array}{l}\text { Tipo de fuente según } \\
\text { clasificación Haynes }\end{array}$} & \multicolumn{2}{|c|}{ Tradicional } & \multicolumn{2}{|c|}{ MBE } & \multirow{2}{*}{ TOTAL } \\
\hline & n & $\%$ & n & $\%$ & \\
\hline Estudios originales & 15 & 5,1 & 130 & 44,1 & \\
\hline Sinopsis de estudios & 0 & 0 & 0 & 0 & \\
\hline Síntesis & 1 & 0,3 & 14 & 4,7 & \\
\hline Sinopsis de síntesis & 1 & 0,3 & 12 & 4,1 & \\
\hline Sumarios o compendios & 31 & 10,6 & 91 & 30,8 & \\
\hline Sistemas Informatizados & 0 & 0 & 0 & 0 & \\
\hline Total & 48 & 16,3 & 247 & 83,7 & 295 \\
\hline
\end{tabular}

Tabla 3. Distribución porcentual del tipo de estudio recuperado por los estudiantes en el método Tradicional y el Método MBE. (n=370).

\begin{tabular}{|c|c|c|c|c|c|}
\hline \multirow{2}{*}{ Tipo de estudio } & \multicolumn{2}{|c|}{ Tradicional } & \multicolumn{2}{|c|}{ MBE } & \multirow{2}{*}{ TOTAL } \\
\hline & $n$ & $\%$ & $n$ & $\%$ & \\
\hline Meta-análisis & 0 & 0 & 6 & 1,6 & \\
\hline Revisión Sistemática & 17 & 4,6 & 14 & 3,8 & \\
\hline Ensayos Clínicos & 1 & 0,3 & 26 & 7,0 & \\
\hline Estudios de cohorte & 0 & 0 & 2 & 0,5 & \\
\hline Estudios de casos y controles & 0 & 0 & 8 & 2,2 & \\
\hline Series de casos & 3 & 0,8 & 19 & 5,2 & \\
\hline Opinión de expertos & 12 & 3,2 & 52 & 14,2 & \\
\hline Guía de prácticas clínicas & 27 & 7,3 & 82 & 22,2 & \\
\hline Textos & 28 & 7,6 & 73 & 19,7 & \\
\hline Total & 88 & 23,6 & 282 & 76,4 & 370 \\
\hline
\end{tabular}


Tabla 4. Estudiantes que identifican referencias según el método tradicional y el Método Basado en Evidencias (N=65).

\begin{tabular}{|c|c|c|c|c|c|c|c|c|c|c|c|c|}
\hline \multirow{3}{*}{$\begin{array}{l}\text { Número de } \\
\text { referencias } \\
\text { identificadas }\end{array}$} & \multicolumn{4}{|c|}{ Tipo de estudio } & \multicolumn{4}{|c|}{ Clasificación Haynes } & \multicolumn{4}{|c|}{ Calidad de evidencia } \\
\hline & \multicolumn{2}{|c|}{ Tradicional } & \multicolumn{2}{|c|}{ MBE } & \multicolumn{2}{|c|}{ Tradicional } & \multicolumn{2}{|c|}{$\mathrm{MBE}$} & \multicolumn{2}{|c|}{ Tradicional } & \multicolumn{2}{|c|}{ MBE } \\
\hline & $\mathbf{N}$ & $\%$ & $\mathbf{N}$ & $\%$ & $\mathbf{N}$ & $\%$ & $\mathbf{N}$ & $\%$ & $\mathbf{N}$ & $\%$ & $\mathbf{N}$ & $\%$ \\
\hline Ninguna & 23 & 35 & 13 & 20 & 36 & 55 & 15 & 23 & 30 & 46 & 12 & 18 \\
\hline de 1 a 3 & 37 & 57 & 13 & 20 & 28 & 43 & 27 & 41 & 28 & 43 & 17 & 26 \\
\hline de 4 a 6 & 5 & 8 & 21 & 32 & 1 & 2 & 9 & 14 & 6 & 9 & 18 & 28 \\
\hline más de 6 & 0 & 0 & 18 & 28 & 0 & 0 & 14 & 22 & 1 & 2 & 18 & 28 \\
\hline Total & 65 & 100 & 65 & 100 & 65 & 100 & 65 & 100 & 65 & 100 & 65 & 100 \\
\hline
\end{tabular}

Tabla 5. Distribución porcentual de los Problemas Relacionados con los Medicamentos (PRM) de efectividad y seguridad, que identificaron los estudiantes capacitados con el método Tradicional y el Método MBE.

\begin{tabular}{|c|c|c|c|c|c|c|c|c|c|c|c|c|}
\hline \multirow{3}{*}{ PRM } & \multicolumn{4}{|c|}{ Tipo de estudio } & \multicolumn{4}{|c|}{ Clasificación Haynes } & \multicolumn{4}{|c|}{ Calidad de evidencia } \\
\hline & \multicolumn{2}{|c|}{ Tradicional } & \multicolumn{2}{|c|}{ MBE } & \multicolumn{2}{|c|}{ Tradicional } & \multicolumn{2}{|c|}{$\mathrm{MBE}$} & \multicolumn{2}{|c|}{ Tradicional } & \multicolumn{2}{|c|}{$\mathrm{MBE}$} \\
\hline & $\mathbf{N}$ & $\%$ & $\mathbf{N}$ & $\%$ & $\mathbf{N}$ & $\%$ & $\mathbf{N}$ & $\%$ & $\mathbf{N}$ & $\%$ & $\mathbf{N}$ & $\%$ \\
\hline Correcto & 14 & 48 & 15 & 52 & 142 & 39 & 221 & 61 & 156 & 40 & 236 & 60 \\
\hline Incorrecto & 20 & 54 & 17 & 46 & 31 & 51 & 30 & 49 & 51 & 52 & 47 & 48 \\
\hline Total & 34 & 52 & 32 & 48 & 173 & 41 & 251 & 59 & 207 & 42 & 283 & 58 \\
\hline
\end{tabular}

base de datos Cochrane; sólo $23 \%$ visitó el sitio de la Biblioteca Virtual durante los últimos 6 meses. En nuestro país, Lara y Miyahira (21) describieron las características del uso de internet por 192 estudiantes del internado de medicina de cuatro universidades de Lima Metropolitana, determinando que el acceso fue $80,2 \%$ desde su casa, $26,6 \%$ desde cabinas públicas, $21,9 \%$ desde la universidad y 10,9\% desde el hospital. El 50,5\% usaba

observó que con la MBE fue mayor, $55 \%$ frente a $45 \%$ con el método tradicional (tabla 6); esto demuestra que al mejorar las competencias para identificar PRM mejora la intervención farmacéutica en un caso clínico seleccionado.

En un estudio realizado en España ${ }^{(18)}$, sugieren un algoritmo de búsqueda de literatura de medicamentos por internet, que tiene vigencia y ayuda a orientar la búsqueda, desde un punto de vista general del medicamento, el cual se complementa con la búsqueda por MBE propuesto en el presente estudio.

Silva-Castro, et al. ${ }^{(19)}$ desarrollaron una encuesta a 76 farmacéuticos que asistieron al III Congreso de Atención Farmacéutica en Granada, para conocer cuáles eran las páginas web más utilizadas para la búsqueda de información sobre Atención Farmacéutica. Estas fueron: BOT, Pharmaceutical Care España, PubMed, Grupo de Investigación de la Universidad de Granada y el CADIME, siendo preferidas las que se publican en españolylas biomédicas, los boletinesdemedicamentos, y las que contienen guías de práctica clínica y revisiones.

González y Santana (20), estudiaron el comportamiento de 60 estudiantes del sexto año de medicina que utilizaron el servicio de navegación en internet en la Biblioteca Médica Nacional de Cuba, encontrando que $80 \%$ de ellos no consultan o desconocen PubMed, Medline y sólo 3\% emplea el Mesh Browser; 95\% de los estudiantes no emplean la
Tabla 6. Distribución porcentual de las Intervenciones Farmacéuticas que elaboran los estudiantes capacitados con el método Tradicional y el Método MBE. ( $\mathrm{n}=329)$.

\begin{tabular}{|c|c|c|c|c|c|}
\hline \multirow{2}{*}{ Intervención Farmacéutica } & \multicolumn{2}{|c|}{ Tradicional } & \multicolumn{2}{|c|}{ MBE } & \multirow{2}{*}{ TOTAI } \\
\hline & n & $\%$ & n & $\%$ & \\
\hline Plantea educación al paciente & 52 & 49 & 55 & 51 & 107 \\
\hline Plantea sugerencia al equipo de salud & 101 & 45 & 121 & 55 & 222 \\
\hline Total & 153 & 46 & 176 & 54 & 329 \\
\hline
\end{tabular}




\section{REFERENCIAS BIBLIOGRÁFICAS}

1. Cipolle RJ, Strand LM, Morley PC. Pharmaceutical care practice. $2^{\text {nd }}$ ed. New York: McGraw-Hill; 2008.

2. Provers JP, Currie JD, Phagel HP, McDonough RP, Sobodka JL. A practical guide to pharmaceutical care. $2^{\text {nd }}$ ed. Washington DC: APhA; 2003.

3. Bradley P, Oterholt C, Herrin J, Nordheim L, Bjørndal A. Comparison of directed and self-directed learning in evidence-based medicine: a randomised controlled trial. Medical Education. 2005; 39(10): 1027-35.

4. Abu-Ramaileh AM, Shane R, Churchill W, Steffenhagen A, et al. Evaluating and classifying pharmacists' quality interventions in the emergency department. Am J Health Syst Pharm. 2011; 68(23): 2271-5.

5. Shuval k, Berkovits E, Netzer D, Hekselman I, Linn S, Brezis $\mathrm{M}$, et al. Evaluating the impact of an evidencebased medicine educational intervention on primary care doctors' attitudes, knowledge and clinical behaviour: a controlled trial and before and after study. Journal of Evaluation in Clinical Practice. 2007; 13(4): 581-98.

6. Organización Panamericana de Salud; Ministerio de Salud del Perú. Selección de medicamentos esenciales. ${ }^{\text {ra }}$ ed. OPS - DIGEMID. Lima; 2010.

7. Burke JM, Miller WA, Spencer AP, Crank CW, et al. ACCP Clinical Pharmacist Competencies. Pharmacotherapy. 2008; 28(6): 806-15

8. Salinitri FD, O'Connell MB, Garwood CL, Lehr VG, et al. An Objective Structured Clinical Examination to Assess Problem-Based Learning. Am J Pharm Educ. 2012; 76(3): 44.

9. Weng YH, Kuo KN, Yang CY, Liao HH, et al. Effectiveness of national evidence-based medicine competition in Taiwan. BMC Medical Education. 2013; 13(66): 1-9.

10. Yan XF, Ni Q, Wei JP, Xu H. Evidence-based practice method of integrative chinese and western medicine based on literature retrieval through PICO question and complementary and alternative medicine topics. Chin J Integr Med. 2010; 16(6): 542-8.

11. Delgado DG, Carreño QR, Cortez MW. Experiencias de una década de Seguimiento Farmacoterapéutico a pacientes de la comunidad en una universidad pública. Ciencia e Investigación. 2012; 15(2): 61-5.

12. Oller GJ. Elementos teórico-prácticos útiles para comprender el uso de los motores de búsqueda en Internet. ACIMED [Internet]. 2003 [Citado 17 dic 2012]; 11(6). Disponible en: http://scielo.sld.cu/scielo. php?pid=S1024-94352003000600007\&script=sci_arttext)

13. Elizondo LG. Recursos disponibles para la recuperación de información sobre salud en Internet. Rev Costarric Salud Pública [Internet]. 2005 [Citado 17 dic 2012]; 14(27). Disponible en: http://www.scielo. sa.cr/scielo.php?script=sci_arttext\&pid=S140914292005000200003\&lang=pt

14. Meats E, Heneghan C, CrillY M, Glasziou P. Evidencebased medicine teaching in UK medical schools. Medical Teacher. 2009; 31(4): 332-7.

15. Thangaratinam S, Barnfield G, Weinbrenner S, Meyerrose $\mathrm{B}$, et al. Teaching trainers to incorporate evidence-based medicine (EBM) teaching in clinical practice: the EUEBM project. BMC Medical Education. 2009; 9: 59.

16. Schardt C, Adams MB, Owens T, Keitz S, et al. Utilization of the PICO framework to improve searching PubMed for clinical questions. BMC Medical Informatics and Decision Making. 2007; 7: 16.

17. Alahdab F, Firwana B, Hasan R, Sonbol MB, et al. Undergraduate medical students' perceptions, attitudes, and competencies in evidence-based medicine (EBM), and their understanding of EBM reality in Syria. BMC Research Notes. 2012; 5: 431.

18. Tordera BM, Magraner JG, Fernández MJ. Información de medicamentos e Internet. Estrategias de búsqueda Farmacoterapéutica en la world wide web. Farmacia Hospitalaria [Internet]. 1999 [Citado 17 dic 2012]; 23(1): 1-13. Disponible en: http://www.elsevier.es/es-revistafarmacia-hospitalaria-121-articulo-informacin-demedicamentos-e-internet--13005170

19. Silva-Castro MM, Bermúdez-Tamayo C, García GJ, Jiménez PJ, et al. Recursos web utilizados por los farmacéuticos para realizar atención farmacéutica. Seguim Farmacoter. 2004; 2(1): 19-23.

20. González MR, Santana SA. Comportamiento de los estudiantes de medicina en la búsqueda de información en Internet. ACIMED. 2008; 17(4).

21. Lara VK, Miyahira AJ. Uso de internet por estudiantes del internado de medicina en Lima Metropolitana. Rev Med Hered. 2009; 20(3).

22. Bookstaver B, Rudisill CN, Bickley AR, McAbee C, et al. An evidence-based medicine elective course to improve student performance in advanced pharmacy practice experiences. Am J Pharm Educ. 2011; 75(1): 9.

Manuscrito recibido el: 02/12/14

Aceptado para su publicación el: 25/05/2015

\section{Correspondencia:}

Nombre: $\quad$ Gladys M. Delgado Pérez.

Dirección: Jr. Puno 1002. Lab. Farmacología

E-mail: $\quad$ gladymarpe@yahoo.com 